\title{
Investigating the Influence of Rois Selection in Breast Ultrasound Segmentation Using the Eicamm Technique
}

\author{
Karem D Marcomini* \\ Department of Electrical Engineering -EESC-University of São Paulo, Brazil
}

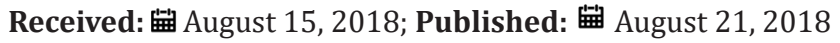

*Corresponding author: Karem D Marcomini, Department of Electrical Engineering -EESC-University of São Paulo, São Carlos (SP), Brazil

\begin{abstract}
The false-negative interpretation represents serious problems in breast lesions diagnosis. In order to reduce the number of these cases and increase the diagnostic sensibility, computational tools have been developed to aid the early detection of breast cancer. However, such computer schemes can be influenced directly or indirectly by the user mainly regarding the selection of the type of image to be processed. In this context, this work evaluates how the non-standardization in cutting regions of interest (ROIs) in the image can affect the computed detection and computer segmentation step. A total of 54 lesions recorded in images from breast ultrasonography were used for the tests. An experienced radiologist cropped each lesion three times varying the amount of surrounding tissue-three different sets were formed, and a test group was added to the study containing 18 lesions of each case selected. A previous developed segmentation procedure based on the use of the EICAMM technique was applied to the images. The most accurate result with the EICAMM technique was obtained in the first set, in which the clipping was made as close to the lesion, providing greater accuracy in the comparison between the segmentation by the computational process and the lesion delineation by the radiologist with lower rates of over and under segmentation.
\end{abstract}

Keywords: Breast Ultrasound Images; Visual Subjectivity; Nodules Segmentation; Eicamm; Computer Aided Detection

Abbrevations: ROIs: Regions Of Interest; CAD: Computer Aided Detection; EICAMM: Enhanced Independent Component Analysis Mixture Model; AOM: Area Overlap Measure; AUM: Under Segmentation Measure; AVM: Over Segmentation Measure; CM: Combination Measure; CP: completeness; CR: correctness; Q: Quality; A: Accuracy; Err: Error; FPR: False Positive Ratio

\section{Introduction}

Mammography is the best method for early detection of breast cancer, and its interpretation remains a challenge to the specialist [1]. However, in women with dense breast, the mammography sensitivity may be low, allowing to miss about $10 \%$ of all cancers [2-3]. Breast ultrasonography has emerged as an important adjunct to diagnostic mammography and it has been used to distinguish between mammographically identified cystic and solid masses. Some problems in diagnosis accuracy are related to miss of lesions, being possible causes: dense parenchyma obscuring a lesion, poor positioning, noisy nature of images, perception error, incorrect interpretation of a suspect finding, subtle features of malignancy, and slow growth of a lesion [4]. In this sense, about $10-30 \%$ of breast lesions are missed in routine exam due to limitations of human observers [1]. With the advance of digital technology, mainly of the digital image processing -including pattern recognition and artificial intelligence-radiologists have the opportunity to improve the diagnostic accuracy with the aid of computer systems. Computer Aided-Detection (CAD) is a relatively new technology which has been implemented in some mammography centers with the purpose of providing double reading, working as a second opinion. CAD schemes are useful when there is high interobserver variability, absence of trained observers or impossibility of performing double reading with two or more radiologists. Clinical studies have demonstrated that CAD increases sensitivity in the detection of breast cancer by radiologists in up to $20 \%$ [1]

Some CAD schemes allow the user interaction in performing particular procedures. For such processes the interobserver variability becomes a problem, which in most cases is related to the lack of standardization to do these tasks. One of them is associated to the selection of the region of interest (ROI), because the way a particular lesion is determined has direct influence on the system output. Therefore, this feature can change the lesion segmentation and hence its classification- highly dependent on the result coming from the previous procedure [5]. Therefore, here the ROI selection effect on these two steps is investigated, by using a novel methodology for masses segmentation based on the Enhanced Independent Component Analysis Mixture Model (EICAMM) [6-7]. Also this work proposes a study of how the lack of standardization for the manual ROIs selection may affect the automatic detection process in breast ultrasound procedures. 


\section{Materials and Methods}

\section{Database}

For the investigation 54 breast ultrasound images containing suspicious lesions were selected. These images were obtained at Diagnosis Imaging Integrated Center-Santa Casa Hospital in Sao Carlos, SP, Brazil. The acquisition was provided by a Siemens G50 equipment, operating with linear array transducer of 7.5-Megahertz (MHz) B-mode. An experienced radiologist carried out the manual contour delineation of the lesions by using Wacom Cintiq 13 HD graphical monitor with digital pen which provides high accuracy, easy and fast manual design, pressure sensitivity and tilt recognition. Thereafter this same radiologist was instructed to perform three different selections for the same lesion considering the follow categories:

a) a cut with plenty of tissue adjacent to the lesion;

b) an intermediate amount of surrounding tissue; and

c) a cut as close as possible to the lesion.

In order to study how the lack of standardization in performing the cut can influence the computational segmentation, we create a differentiated group containing 18 images of each of the three sets a total of 54 ROIs, so that each lesion were included only once. This group was called Test Group.

\section{Digital Processing}

The EICAMM method [6] is based on adaptations of the Independent Component Analysis Mixture Model (ICAMM) technique [7], which incorporated improvements in some aspects of nonlinear optimization in order to overcome some limitations of this latter [7]. Some of the major changes are: formulation of a more informative learning rule for the element bias from the approach to maximize the mutual information that the processor output of the neural network has relative to the input (the network takes into account, in the current iteration, the results obtained in previous iterations); regarding the convergence acceleration of the algorithm and the assurance of the local minimum of the function, the update rule was modified by incorporating the second derivative, using the methods of Newton and Levenberg-Marquardt; orthogonalization of the base matrix [6-8]. EICAMM model considers that the points to be clustered are generated by a mathematical process described as a mixture of $\mathrm{k}$ probability densities classes.

In this way, the aim of the clustering is to find the parameters of each class distribution and assign each sample according to the highest probability [7]. The input data X corresponds to the coordinates of each pixel of the original images and its neighbors, as exemplified in Figure 1. Convolution is used to indicate the value of the neighborhood of pixels located at the edge of the image [7]. For the convolution, Ribeiro [6] choose to take the values of the pixel edges, for reducing possible errors edge information with neighborhood of size $3 \times 3$. After segmentation, morphological operations of opening and closing are applied for smoothing the edges and removing some erroneously connected pixels that joined the lesion and the background. Such a procedure was validated by using another segmentation technique that provided similar results as to noisy appearance of the contour, described by Marcomini [9]. Then a new operation was performed on the image, the internal valleys ("Holes") were added to the segmented region and the unconnected areas to the object of interest were eliminated [10].

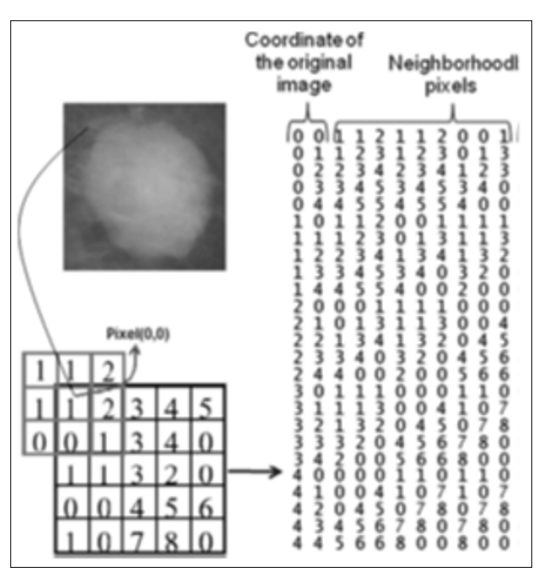

Figure 1: Matrix input for EICAMM.

\section{Results}

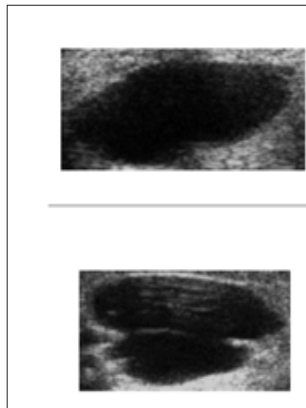

(a)
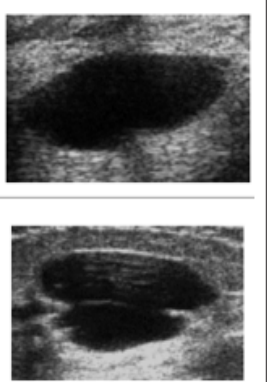

(b)

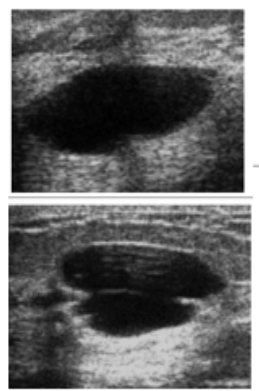

(c)

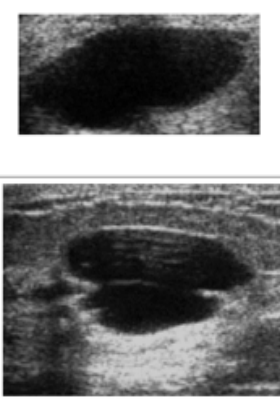

(d)
Figure 2: ROIs from breast ultrasound images. (a) Class 1; (b) Class 2; (c) Class 3; (d) Test Group.

The crop size and a standard criterion for its performance are extremely important to the lesions detection in medical images. The variations in the gray scale involve changes in the mathematical calculations which aim to cluster similar regions and consequently differentiate the object of interest from the background. In automatic processes this influence is even greater because the initial parameters of the segmentation algorithm are not adjusted according to the characteristics of the input image. With this aim 
three sets of ROIs cropped by the radiologist and a test set were created (Figure 2) illustrates some examples of the 4 cases. The new segmentation technique EICAMM was then used to demonstrate that accurate detection of lesions in breast ultrasound images is related to the size of the ROI. The segmentation accuracy was evaluated by comparing the results from the images processing with the manual delineation performed by an experienced radiologist. This comparative was quantified through ten measures which were described by Marcomini [9], which are: area overlap measure (AOM), under segmentation measure (AUM), over segmentation measure (AVM), combination measure (CM), completeness (CP), correctness (CR), quality (Q), accuracy (A), error (Err), and false positive ratio (FPR), whose ideal values are approaching the required value. The percentage mean values obtained with each class ROI are listed in Table 1.

Table 1: Evaluation metrics applied to breast ultrasound images segmentation.

\begin{tabular}{|c|c|c|c|c|c|c|c|c|c|c|}
\hline & AOM (\%) & AUM (\%) & AVM (\%) & CM (\%) & CP (\%) & CR (\%) & Q (\%) & A (\%) & Err & FPR \\
\hline Class 1 & 66.31 & 29.77 & 6.09 & 76.81 & 70.23 & 93.91 & 66.31 & 82.26 & 14.74 & 2.76 \\
\hline Class 2 & 46.23 & 37.46 & 44.80 & 54.65 & 62.54 & 55.20 & 46.23 & 85.82 & 14.18 & 9.82 \\
\hline Class 3 & 20.54 & 66.46 & 76.09 & 26.00 & 33.54 & 23.91 & 20.54 & 82.15 & 17.85 & 13.74 \\
\hline Class 4 & 44.87 & 47.23 & 42.90 & 51.59 & 52.77 & 57.10 & 44.87 & 84.83 & 15.17 & 8.49 \\
\hline Required Value & 100 & 0 & 0 & 100 & 100 & 100 & 100 & 100 & 0 & 0 \\
\hline
\end{tabular}

The main reason of inaccuracy in many CAD schemes is related to the incorrect detection in the morphology and contour of the lesion, especially when it comes from an automatic system. This is even worse in ultrasound imaging due to the noisy look of the image. To minimize this problem, other smoothing techniques should be tested in future studies in order to increase the accuracy of segmentation and consequently the similarity between automatic and the radiologist visual detections. Thereby the feasibility of using the EICAMM technique in breast ultrasound images segmentation could be improved. A further limitation to be overcome is the high computational cost to perform the classes clustering. Non-standardization of user interaction has also a significant impact on the segmentation accuracy. This feature is enhanced by measurements obtained from the comparison between the automatic segmentation and that determined by the radiologist.

The most accurate among the four classes considered here was the first - where the cutting to select the ROI to be segmented is performed as close as possible to the lesion, which yields the smallest effect of the background on the segmentation. When the clipping includes a greater amount of surrounding tissue, both under (AUM) and over-segmentation (AVM) are increased, taking to the detection of erroneous regions. The set with random clippings (4) provided intermediate rates, emphasizing the need for standardization to ensure the result accuracy. Even though the cut for ROI selection can be subjective and radiologist dependent, criteria should be established for its determination so that the algorithm get desirable results. For this case, it should include low amount of surrounding tissue, but taking care that the entire lesion be included in the cut.

\section{References}

1. Calas MJG, Gutfilen B, Pereira WCA (2012) CAD and mammography: why use this tool? Radiologia Brasileira 45(1): 46-52.

2. Berg WA, Blume JD, Cormack JB, Mendelson EB, Lehrer D, et al. (2008) Combined screening with ultrasound and mammography vs mammography alone in women at elevated risk of breast cancer. Journal of the American Medical Association 299(18): 2151-2162.

3. Sivaramakrishnaa R, Powella KA, Lieberb ML, Chilcotec WA, Shekhara $R$ (2002) Texture analysis of lesions in breast ultrasound images. Computerized Medical Imaging and Graphics 26(5): 303-307.

4. Devolli Disha E, Manxhuka Kerliu S (2009) Comparative accuracy of mammography and ultrasound in women with breast symptoms according to age and breast density. Bosnia Journal Basic Medical Science 9(2): 131-136.

5. Gruszauskas NP, Drukker K, Giger M, CA Sennett M, Pesce LL (2008) Performance of breast ultrasound computer aided-diagnosis: Dependence on image selection. Academic Radiology 15(10): 12341245.

6. Ribeiro PB, Romero RAF, Oliveira PR, Schiabel H, Verçosa LB (2013) Automatic segmentation of breast masses using enhanced ICA mixture model. Neurocomputing 120: 61-71.

7. Oliveira PR, Romero RAF (2004) Enhanced ICA mixture model for image segmentation. International Conference on Machine Learning and Applications (ICMLA), Louisville, USA.

8. Oliveira PR, Romero RAF (2008) Improvements on ICA mixture models for image pre-processing and segmentation. Neurocomputing 71(10): 2180-2193.

9. Marcomini KD, Schiabel H, Carneiro AAO (2013) Quantitative evaluation of automatic methods for lesions detection in breast ultrasound images. Proc, USA, 8670: 271-277.

10. Marcomini KD, Schiabel H (2012) Nodules segmentation in breast ultrasound using the artificial neural network Self-Organizing Map. Proc of World Congress on Engineering, London 2: 1152-1155. 
ISSN: 2574-1241

DOI: 10.26717/BJSTR.2018.08.001617

Karem D Marcomini. Biomed J Sci \& Tech Res

(c) (P) This work is licensed under Creative

Submission Link: https://biomedres.us/submit-manuscript.php

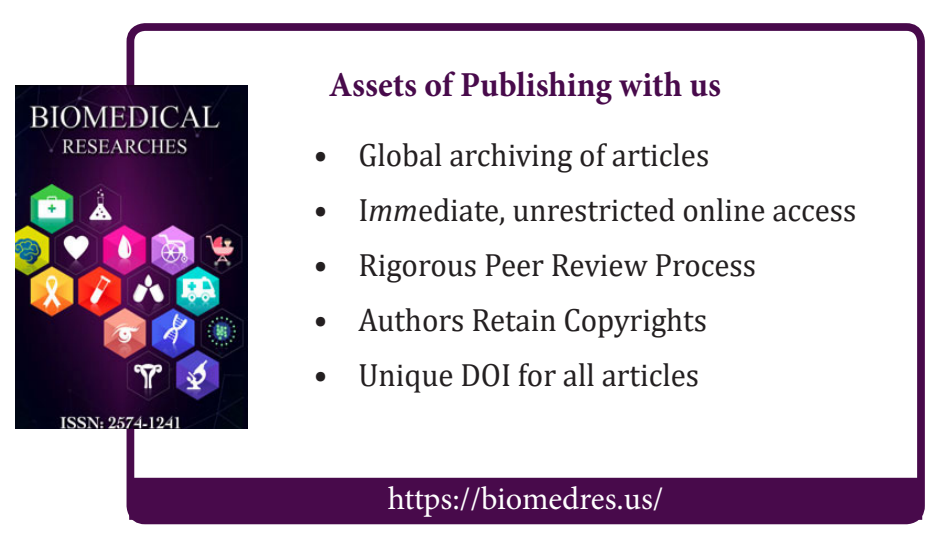

